\title{
A matrix recurrence for systems of Clifford algebra-valued orthogonal polynomials
}

\author{
I. Cação ${ }^{a}$ \\ M.I. Falcão ${ }^{b}$ \\ H.R. Malonek ${ }^{a}$ \\ ${ }^{a}$ Departamento de Matemática and CIDMA, Universidade de Aveiro, Portugal \\ ${ }^{b}$ Departamento de Matemática e Aplicações and CMAT, Universidade do Minho, Portugal
}

\begin{abstract}
Recently, the authors developed a matrix approach to multivariate polynomial sequences by using methods of Hypercomplex Function Theory (Matrix representations of a basic polynomial sequence in arbitrary dimension. Comput. Methods Funct. Theory, 12 (2012), no. 2, 371-391). This paper deals with an extension of that approach to a recurrence relation for the construction of a complete system of orthogonal Clifford-algebra valued polynomials of arbitrary degree. At the same time the matrix approach sheds new light on results about systems of Clifford algebra-valued orthogonal polynomials obtained by Gürlebeck, Bock, Lávička, Delanghe et al. during the last five years. In fact, it allows to prove directly some intrinsic properties of the building blocks essential in the construction process, but not studied so far.
\end{abstract}

\section{Information}

Keywords:

Clifford Analysis; generalized Appell polynomials; recurrence relations.

Original publication:

Adv. Appl. Clifford Algebra 24, (2014), 981- 994

DOI: $10.1007 /$ s00006-014-0505-x

http://link.springer.com

Dedicated to Professor Klaus Gürlebeck on the occasion of his 60th birthday

\section{Introduction}

\subsection{Preliminaries}

Approaches to multivariate polynomial sequences by methods of Hypercomplex Function Theory are usually based on the following facts (see e. g. $[4,20]$ ). Let $\left\{e_{1}, e_{2}, \ldots, e_{n}\right\}$ be an orthonormal basis of the Euclidean vector space $\mathbb{R}^{n}$ with a non-commutative product according to the multiplication rules

$$
e_{k} e_{l}+e_{l} e_{k}=-2 \delta_{k l}, \quad k, l=1, \ldots, n,
$$

where $\delta_{k l}$ is the Kronecker symbol. The set $\left\{e_{A}: A \subseteq\{1, \ldots, n\}\right\}$ with

$$
e_{A}=e_{h_{1}} e_{h_{2}} \ldots e_{h_{r}}, \quad 1 \leq h_{1}<\cdots<h_{r} \leq n, \quad e_{\emptyset}=e_{0}=1,
$$

forms a basis of the $2^{n}$-dimensional Clifford algebra $\mathcal{C} \ell_{0, n}$ over $\mathbb{R}$. Let $\mathbb{R}^{n+1}$ be embedded in $\mathcal{C} \ell_{0, n}$ by identifying $\left(x_{0}, x_{1}, \ldots, x_{n}\right) \in \mathbb{R}^{n+1}$ with

$$
x=x_{0}+\underline{x} \in \mathcal{A}_{n}:=\operatorname{span}_{\mathbb{R}}\left\{1, e_{1}, \ldots, e_{n}\right\} \subset \mathcal{C} \ell_{0, n} .
$$

Here, $x_{0}=\operatorname{Sc}(x)$ and $\underline{x}=\operatorname{Vec}(x)=e_{1} x_{1}+\cdots+e_{n} x_{n}$ are the scalar and vector parts of the paravector $x \in \mathcal{A}_{n}$. The conjugate of $x$ is given by $\bar{x}=x_{0}-\underline{x}$ and its norm by $|x|=(x \bar{x})^{\frac{1}{2}}=\left(x_{0}^{2}+x_{1}^{2}+\cdots+x_{n}^{2}\right)^{\frac{1}{2}}$. 
To call attention to its relation to the complex Wirtinger derivatives, we use the following notation for a generalized Cauchy-Riemann operator in $\mathbb{R}^{n+1}, n \geq 1$,

$$
\bar{\partial}:=\frac{1}{2}\left(\partial_{0}+\partial_{\underline{x}}\right)
$$

and its conjugate

$$
\partial:=\frac{1}{2}\left(\partial_{0}-\partial_{\underline{x}}\right)
$$

with

$$
\partial_{0}:=\frac{\partial}{\partial x_{0}} \quad \text { and } \quad \partial_{\underline{x}}:=e_{1} \frac{\partial}{\partial x_{1}}+\cdots+e_{n} \frac{\partial}{\partial x_{n}} .
$$

$\mathcal{C}^{1}$-functions $f$ satisfying the equation $\bar{\partial} f=0$ (resp. $f \bar{\partial}=0$ ) are called left monogenic (resp. right monogenic). We suppose that $f$ is hypercomplex-differentiable in $\Omega$ in the sense of [19, 24], that is, it has a uniquely defined areolar derivative $f^{\prime}$ in each point of $\Omega$ (see also [25]). Then, $f$ is real-differentiable and $f^{\prime}$ can be expressed by the conjugate generalized Cauchy-Riemann operator as $f^{\prime}=\partial f$. Since a hypercomplex differentiable function belongs to the kernel of $\bar{\partial}$, it follows that, in fact, $f^{\prime}=\partial_{0} f=-\partial_{\underline{x}} f$ which is similar to the complex case.

Needless to mention that $\mathcal{C} \ell_{0, n}$-valued functions defined in some open subset $\Omega \subset \mathbb{R}^{n+1}$ are of the form $f(z)=\sum_{A} f_{A}(z) e_{A}$ with real valued $f_{A}(z)$.

We use also the classical definition of sequences of Appell polynomials [1] adapted to the hypercomplex case.

Definition 1.1 A sequence of homogeneous monogenic polynomials $\left(\mathcal{F}_{k}\right)_{k \geq 0}$ of exact degree $k$ is called a generalized Appell sequence with respect to $\partial$ if

1. $\mathcal{F}_{0}(x) \equiv 1$,

2. $\partial \mathcal{F}_{k}=k \mathcal{F}_{k-1}, k=1,2, \ldots$

The second condition is the essential one while the first condition is the usually applied normalization condition which can be changed to any real or hypercomplex constant different from zero or even to a generalized constant. A generalized constant is a monogenic function whose hypercomplex derivative is zero. In dimension three $(n=2)$ a generalized constant is isomorphic to an anti-holomorphic function as referred in [12].

Due to our goal of a matrix recurrence for orthogonal polynomials, we need also the Clifford algebra-valued inner product

$$
(f, g)_{\mathcal{C} \ell_{0, n}}=\int_{B^{n+1}} \bar{f} g d \lambda^{n+1},
$$

being $\lambda^{n+1}$ the Lebesgue measure in $\mathbb{R}^{n+1}$ and $\bar{a}$ the conjugate of $a \in \mathcal{C} \ell_{0, n}$.

Finally we notice that several authors $([16,23])$ prefer to consider monogenic functions as solutions of the Dirac equation

$$
\partial_{\underline{x}} f=0
$$

in the Euclidean space $\mathbb{R}^{n}, n \geq 1$, and not as solutions of a generalized Cauchy-Riemann system like in our case. The essential difference to our approach in the context of Appell sequences consists in the fact that among all real variables $x_{k}, k=1 \ldots n$, a-priori does not exist an exceptional variable like $x_{0}$ that could serve as reference for a hypercomplex derivative as explained before. Therefore they use, in general, the last variable $x_{n}$ and rely their explanations on the fundamental concept of Fischer decompositions for harmonic, respectively monogenic polynomials with respect to $\underline{x} e_{n}$ instead of $\underline{x}$. In this case the variable $x_{n}$ plays the role of an exceptional variable and the hypercomplex derivative $\partial f:=\frac{1}{2}\left(\partial_{0}-\partial_{\underline{x}}\right) f$ is substituted by the real partial derivative $\partial_{x_{n}} f$. Of course, the difference of both approaches seems to be not essential. Nevertheless, the fact that also the Cauchy-Kovalevskaya extension $([4,15])$ of polynomials defined in $\mathbb{R}^{n-1}$ to monogenic polynomials in $\mathbb{R}^{n}$ comes into the play complicates the natural inclusion of the real $(\underline{x}=0)$ or complex case $(n=1)$. Working in the framework of monogenic functions as solutions of a generalized Cauchy-Riemann system avoids this problem (see Section 2). 


\subsection{Motivation}

The mathematical interests of Klaus Gürlebeck cover a wide range from theoretical to numerical aspects in Clifford Analysis. For several years, one of the main subjects in the study of monogenic functions by Klaus Gürlebeck and his disciples and collaborators was the construction of orthonormal polynomial systems in the $L^{2}$-space of monogenic Clifford algebra-valued functions in the unit ball of $\mathbb{R}^{n+1}$. As examples, we mention here only $[5,6,7,11])$, where, mainly for practical applications, the case $n=2$ was studied in great detail, by different approaches, and from different points of view.

But the breakthrough to a new and elegant approach came with the paper [2], where S. Bock and K. Gürlebeck succeeded to determine an Appell system as orthogonal basis in the space of square integrable monogenic quaternion-valued functions ${ }^{1}$. Their methods relied on a process of monogenic primitivation for constructing the Appell system. It was the connection of demanding orthogonality together with being of Appell type that gave their result a particular elegance. The paper [22] confirmed their results from a representation theoretical point of view followed by a more general and extensive explanation in the joint work [3] referring to the role of Gelfand-Tsetlin bases.

Recent results of our own research (see [10]) and the analysis of the aforementioned papers showed that a matrix approach to Appell sequences can also advantageously be used.

The results in [3] were later generalized in a systematic way in the paper [23] where the author constructed Gelfand-Tsetlin bases for Clifford-valued homogeneous solutions of the Dirac operator in arbitrary dimensional Euclidean space.

Reformulating these general results in terms of the generalized Cauchy-Riemann operator, we extend in Section 3 our approach to the building blocks that are essential in the construction of a complete system of orthogonal polynomials. The deduced matrix recurrence relation additionally allows to prove some of their intrinsic properties in Section 4.

\section{A Clifford algebra-valued orthogonal system}

In [18] the construction of an orthogonal basis for the space $\mathcal{H}_{k}\left(\mathbb{R}^{n+1}\right)$ of homogeneous harmonic polynomials of degree $k$ relies on the following decomposition

$$
\mathcal{H}_{k}\left(\mathbb{R}^{n+1}\right)=\bigoplus_{j=0}^{k} F_{n+1, j}^{(k-j)} \mathcal{H}_{j}\left(\mathbb{R}^{n}\right)
$$

orthogonal with respect to the usual real-valued $L^{2}$-inner product in the unit ball $B^{n+1}$ of $\mathbb{R}^{n+1}$. The embedding factors $F_{n+1, j}^{(k-j)}$ are defined as the polynomials

$$
F_{n+1, j}^{(k-j)}(x)=\frac{(j+1)_{k-j}}{(n-1+2 j)_{k-j}}|x|^{k-j} C_{k-j}^{\frac{n-1}{2}+j}\left(\frac{x_{0}}{|x|}\right), \quad x \in \mathbb{R}^{n+1},
$$

where $x=\left(x_{0}, \ldots, x_{n}\right),|$.$| stands for the usual Euclidean norm in \mathbb{R}^{n+1}$, the raising factorial $(\mu)_{m}=$ $\mu(\mu+1)(\mu+2) \cdots(\mu+m-1)$ is represented by the Pochammer symbol $(.)_{m}$ and $C_{m}^{\nu}$ is the Gegenbauer polynomial given by

$$
C_{m}^{(\nu)}(t)=\sum_{i=0}^{\lfloor m / 2\rfloor}(-1)^{i} \frac{(\nu)_{m-i}}{i !(m-2 i) !}(2 t)^{m-2 i}, \quad t \in \mathbb{R}, \quad \nu \neq 0 .
$$

It is well-known that the Gegenbauer polynomials $C_{m}^{(\nu)}$ satisfy several recurrence relations as e.g.:

$$
\begin{gathered}
\frac{d}{d t} C_{m}^{(\nu)}(t)=2 \nu C_{m-1}^{(\nu+1)}(t) \\
\left(1-t^{2}\right) \frac{d}{d t} C_{m}^{(\nu)}(t)=(m+2 \nu) t C_{m}^{(\nu)}(t)-(m+1) C_{m+1}^{(\nu)}(t)
\end{gathered}
$$

\footnotetext{
${ }^{1}$ Notice that in case of the classical orthogonal polynomials of one real variable only the Hermite polynomials are also Appell sequences in the sense of [1] as has been shown by W. Hahn in [21] for the first time.
} 


$$
\frac{d}{d t} C_{m+1}^{(\nu)}(t)=(m+2 \nu) C_{m}^{(\nu)}(t)+t \frac{d}{d t} C_{m}^{(\nu)}(t)
$$

An orthogonal decomposition analogous to (2.1) was described in [15] for the space $\mathcal{M}_{k}\left(\mathbb{R}^{n+1}, \mathcal{C} \ell_{0, n}\right)$ of homogeneous monogenic polynomials of degree $k$ with values in the Clifford algebra $C \ell_{0, n}$. Here it is considered the Clifford algebra-valued inner product (1.1).

The described decomposition uses two fundamental tools in Clifford analysis: the Fischer decomposition and the Cauchy-Kovalevskaya extension. In our context, the result can be formulated as the following.

Theorem 2.1 [15] The space $\mathcal{M}_{k}\left(\mathbb{R}^{n+1}, \mathcal{C} \ell_{0, n}\right)$ has the orthogonal decomposition

$$
\mathcal{M}_{k}\left(\mathbb{R}^{n+1}, \mathcal{C} \ell_{0, n}\right)=\bigoplus_{j=0}^{k} X_{n+1, j}^{(k-j)} \mathcal{M}_{j}\left(\mathbb{R}^{n}, \mathcal{C} \ell_{0, n}\right)
$$

The embedding factors $X_{n+1, j}^{(k-j)}$ are defined as the polynomials

$$
X_{n+1, j}^{(k-j)}(x)=F_{n+1, j}^{(k-j)}(x)+\frac{j+1}{n+2 j} F_{n+1, j+1}^{(k-j-1)}(x) \underline{x}, \quad x \in \mathcal{A}_{n},
$$

where $\underline{x}=x_{1} e_{1}+\cdots+x_{n} e_{n}, F_{n+1, j}^{(k-j)}$ are given by (2.2) and $F_{n+1, k+1}^{(-1)} \equiv 0$.

This theorem ensures that homogeneous monogenic polynomials in $\mathbb{R}^{n+1}$ can be expressed in terms of homogeneous monogenic polynomials in $\mathbb{R}^{n}$, i.e., each $\mathcal{P}_{k} \in \mathcal{M}_{k}\left(\mathbb{R}^{n+1}, \mathcal{C} \ell_{0, n}\right)$ can be represented by

$$
\mathcal{P}_{k}(x)=\sum_{j=0}^{k} X_{n+1, j}^{(k-j)}(x) P_{j}(\underline{x})
$$

for some uniquely determined polynomials $P_{j} \in \mathcal{M}_{j}\left(\mathbb{R}^{n}, \mathrm{C}_{0, n}\right)$.

We emphasize that each $P_{j} \in \mathcal{M}_{j}\left(\mathbb{R}^{n}, \mathcal{C} \ell_{0, n}\right)$ does not depend on $x_{0}$ and therefore can be considered as a generalized constant when embedded in the space $\mathcal{M}_{k}\left(\mathbb{R}^{n+1}, \mathcal{C} \ell_{0, n}\right)$.

We remark that the building blocks (2.7) are slightly different from those obtained in [15], where the authors constructed the Cauchy-Kovalevskaya extension of the polynomials $\underline{x}^{k-j} P_{j}(\underline{x})$. Here we consider the Cauchy-Kovalevskaya extension of the scaled polynomials $c_{k, j}\left(\begin{array}{c}k \\ j\end{array}\right) \underline{x}^{k-j} P_{j}(\underline{x})$, where $c_{k, j}$ are real constants, depending on $k$ and $j$, given by

$$
c_{k, j}(n)=\left\{\begin{array}{ll}
\frac{(k-j) ! !(n+2 j-2) ! !}{(n+k+j-1) ! !}, & \text { if } k, j \text { have different parities } \\
c_{k-1, j}(n), & \text { if } k, j \text { have the same parity }
\end{array} .\right.
$$

The consideration of these scaled polynomials ensures that

$$
\partial_{0} X_{n+1, j}^{(k-j)}(x)=k X_{n+1, j}^{(k-1-j)}(x), \text { for all } x \in \mathcal{A}_{n},
$$

which guarantees that, for each fixed $j(j=0, \ldots, k),\left\{X_{n+1, j}^{(k-j)}(x) P_{j}(\underline{x})\right\}_{k \in \mathbb{N}_{0}}$ is an Appell system, i.e.

$$
\partial\left[X_{n+1, j}^{(k-j)}(x) P_{j}(\underline{x})\right]=k X_{n+1, j}^{(k-1-j)}(x) P_{j}(\underline{x}), \quad x \in \mathcal{A}_{n} .
$$

In the case $j=0$, the particular sequence $\left\{X_{n+1,0}^{(k)}(x) P_{0}(\underline{x})\right\}_{k \in \mathbb{N}_{0}}$ coincides with the sequence $\left\{\mathcal{P}_{k}^{n}\right\}_{k \in \mathbb{N}_{0}}$ constructed in $[17,26]$, where the study of paravector-valued Appell sequences in $\mathbb{R}^{n+1}$ was considered for the first time. As a generalization of the classical Appell sequence of one real variable, the polynomials $\mathcal{P}_{k}^{n}$ have the initial value $\mathcal{P}_{0}(x) \equiv 1$ and can be represented by the binomial-type formula

$$
\mathcal{P}_{k}^{n}(x)=\sum_{s=0}^{k}\left(\begin{array}{l}
k \\
s
\end{array}\right) c_{s}(n) x_{0}^{k-s} \underline{x}^{s},
$$

where $c_{s}(n):=c_{s, 0}(n)(s=0, \ldots, k)$ are given by (2.8). We observe that $X_{n+1, k}^{(0)}(x) \equiv 1$, for all $x \in \mathcal{A}_{n}$ and all $k \in \mathbb{N}_{0}$. 
Remark 2.1 This Appell sequence was generalized in [27] by considering as first term an arbitrary generalized polynomial constant of a fixed degree. The resulting sequence, designated by the author as shifted Appell, can be considered equivalent to $\left\{X_{n+1, j}^{(k-j)}(x) P_{j}(\underline{x})\right\}_{k \in \mathbb{N}_{0}}$.

Theorem 2.1 permits to describe a recursive method for building monogenic polynomial bases in arbitrary dimensions starting from a basis in $\mathbb{R}^{2}$ with values in the Clifford algebra $\mathcal{C} \ell_{0,2}$ (see $\left.[23,3]\right)$. Indeed, an orthogonal basis for the space $\mathcal{M}_{k}\left(\mathbb{R}^{n+1}, \mathcal{C} \ell_{0, n}\right)$ is formed by the polynomials

$$
f_{k, \mu}=X_{n+1, k_{n}}^{\left(k-k_{n}\right)} X_{n, k_{n-1}}^{\left(k_{n}-k_{n-1}\right)} \cdots X_{3, k_{2}}^{\left(k_{3}-k_{2}\right)} \zeta^{k_{2}}
$$

where $\zeta:=x_{1}-x_{2} e_{1} e_{2}$ and $\mu$ is an arbitrary sequence of integers $\left(k_{n+1}, k_{n}\right.$, $\left.\ldots, k_{3}, k_{2}\right)$ such that $k=k_{n+1} \geq k_{n} \geq \cdots \geq k_{3} \geq k_{2} \geq 0$. For details on the construction, see [23].

Notice that $\zeta$ and its integer powers $\zeta^{k_{2}},\left(k_{2} \in \mathbb{N}_{0}\right)$ are the lowest degree polynomials in the set (2.9), for fixed $k$. They form an orthogonal system of generalized constants in $\mathcal{M}_{k}\left(\mathbb{R}^{n+1}, \mathcal{C} \ell_{0, n}\right)$.

\section{A matrix recurrence formula}

In [10] a matrix recurrence procedure was obtained to produce the polynomial $\mathcal{P}_{k}^{n}$ from the given polynomial $\mathcal{P}_{k-1}^{n}$ one degree lower. Our aim is to generalize this result for the building blocks of (2.6) that form the orthogonal system $\left\{X_{n+1, j}^{(k-j)} P_{j} ; j=0, \ldots, k\right\}_{k \in \mathbb{N}_{0}}$.

We begin by reformulating the above mentioned result in terms of the real variable

$$
t:=\frac{x_{0}}{|x|} \in[-1,1]
$$

which implies that $\sqrt{1-t^{2}}=\frac{|\underline{x}|}{|x|} \in[-1,1]$.

Using the function $\underline{\omega}(\underline{x})=\frac{\underline{x}}{\mid \underline{|x|}} \in S^{n}$, with $\underline{\omega}^{2}=-1$, the polynomial $\mathcal{P}_{k}^{n}$ can be written in a complex-like form

$$
\mathcal{P}_{k}^{n}(t,|x|)=|x|^{k}\left[u_{k}^{n}(t)+\underline{\omega} v_{k}^{n}(t)\right]
$$

where $u_{k}^{n}$ and $v_{k}^{n}$ are the real-valued polynomials of degree $k$ given by

$$
\left\{\begin{array}{l}
u_{k}^{n}(t)=\sum_{l=0}^{\left\lfloor\frac{k}{2}\right\rfloor}\left(\begin{array}{c}
k \\
2 l
\end{array}\right)(-1)^{l} c_{2 l}(n) t^{k-2 l}\left(1-t^{2}\right)^{l}, k \geq 1 \\
u_{0}^{n}(t)=1
\end{array}\right.
$$

and

$$
\left\{\begin{array}{l}
v_{k}^{n}(t)=\sqrt{1-t^{2}} \sum_{l=1}^{\left.\frac{k+1}{2}\right\rfloor}\left(\begin{array}{c}
k \\
2 l-1
\end{array}\right)(-1)^{l-1} c_{2 l-1}(n) t^{k-2 l+1}\left(1-t^{2}\right)^{l-1}, k \geq 1, \\
v_{k}^{n}(0)=0
\end{array}\right.
$$

where the coefficients $c_{s}(n):=c_{s, 0}(n)(s=0, \ldots, k)$ are given by (2.8).

Using the new representation (3.1), Theorem 5 of [10] can be reformulated as follows:

Theorem 3.1 [10] The real-valued functions $u_{k}^{n}$ and $v_{k}^{n}$ in the representation (3.1) of $\mathcal{P}_{k}^{n}$ satisfy, for each $k \geq 1$,

$$
\left[\begin{array}{l}
u_{k}^{n}(t) \\
v_{k}^{n}(t)
\end{array}\right]=M_{k}^{n}\left[\begin{array}{l}
u_{k-1}^{n}(t) \\
v_{k-1}^{n}(t)
\end{array}\right],
$$

where

$$
M_{k}^{n}:=M_{k}^{n}(t)=\left[\begin{array}{cc}
t & -\sqrt{1-t^{2}} \\
\frac{k}{k+n-1} \sqrt{1-t^{2}} & \frac{k}{k+n-1} t
\end{array}\right]
$$


In order to generalize this result for the polynomials $X_{n+1, j}^{(k-j)}$, we start by representing them in the complexlike form analogous to (3.1). The relation (2.3) satisfied by the Gegenbauer polynomials and the use of the function $\underline{\omega}$ permit to rewrite the polynomials $X_{n+1, j}^{(k-j)}(j=0, \ldots, k)$ as

$$
X_{n+1, j}^{(k-j)}(t,|x|)=|x|^{k-j}\left[u_{k-j, j}^{n}(t)+\underline{\omega} v_{k-j, j}^{n}(t)\right] .
$$

Here $u_{k-j, j}^{n}, v_{k-j, j}^{n}$ are the real-valued polynomials of degree $k-j$, given by

$$
\left\{\begin{array}{l}
u_{k-j, j}^{n}(t)=A_{k, j}^{n}(k+n-1+j) C_{k-j}^{\left(\frac{n-1}{2}+j\right)}(t) \\
v_{k-j, j}^{n}(t)=A_{k, j}^{n} \sqrt{1-t^{2}} \frac{d}{d t} C_{k-j}^{\left(\frac{n-1}{2}+j\right)}(t)
\end{array},\right.
$$

where $A_{k, j}^{n}:=\frac{k !}{j !(n-1+2 j)_{k+1-j}}$, with the convention that $A_{k, 0}^{1}:=1$, for all $k \in \mathbb{H}$.

Thus the generalization of Theorem 3.1 leads to the following:

Theorem 3.2 For each fixed $k$ and $j,(k \geq 1, j=0, \ldots, k-1)$, the polynomial $X_{n+1, j}^{(k-j)}$ of degree $k-j$ can be obtained from the polynomial of the same family one degree lower by the matrix recurrence relation

$$
\left[\begin{array}{l}
u_{k-j, j}^{n}(t) \\
v_{k-j, j}^{n}(t)
\end{array}\right]=M_{k, j}^{n}\left[\begin{array}{l}
u_{k-1-j, j}^{n}(t) \\
v_{k-1-j, j}^{n}(t)
\end{array}\right]
$$

where

$$
M_{k, j}^{n}:=M_{k, j}^{n}(t)=\left[\begin{array}{cc}
\frac{k}{k-j} t & -\frac{k}{k-j} \sqrt{1-t^{2}} \\
\frac{k}{k+n+j-1} \sqrt{1-t^{2}} & \frac{k}{k+n+j-1} t
\end{array}\right] .
$$

Here $u_{k-j, j}^{n}, v_{k-j, j}^{n}$ and $u_{k-1-j, j}^{n}, v_{k-1-j, j}^{n}$ are the components of the polynomials $X_{n+1, j}^{(k-j)}$ and $X_{n+1, j}^{(k-1-j)}$, respectively, in the representation (3.2).

Proof The right-hand side of (3.4) equals to

$$
\left[\begin{array}{c}
\frac{k}{k-j} t u_{k-1-j, j}^{n}(t)-\frac{k}{k-j} \sqrt{1-t^{2}} v_{k-1-j, j}^{n}(t) \\
\frac{k}{k+n+j-1} \sqrt{1-t^{2}} u_{k-1-j, j}^{n}(t)+\frac{k}{k+n+j-1} t v_{k-1-j, j}^{n}(t)
\end{array}\right] .
$$

In view of (3.3) the first row of (3.5) is given by

$$
\begin{aligned}
\frac{k}{k-j} A_{k-1, j}^{n}\left[t(k+n+j-2) C_{k-1-j}^{\left(\frac{n-1}{2}+j\right)}(t)-\right. & \left.\left(1-t^{2}\right) \frac{d}{d t} C_{k-1-j}^{\left(\frac{n-1}{2}+j\right)}(t)\right] \\
& =A_{k-1, j}^{n} k C_{k-j}^{\left(\frac{n-1}{2}+j\right)}(t),
\end{aligned}
$$

where the equality follows from the recurrence formula (2.4).

The second row of (3.5) is given by

$$
\begin{array}{r}
\frac{k}{k+n+j-1} \sqrt{1-t^{2}} A_{k-1, j}^{n}\left[(k+j+n-2) C_{k-1-j}^{\left(\frac{n-1}{2}+j\right)}(t)+t \frac{d}{d t} C_{k-1-j}^{\left(\frac{n-1}{2}+j\right)}(t)\right] \\
=\frac{k}{k+n+j-1} A_{k-1, j}^{n} \sqrt{1-t^{2}} \frac{d}{d t} C_{k-j}^{\left(\frac{n-1}{2}+j\right)}(t),
\end{array}
$$

where the equality follows from the recurrence formula (2.5).

Taking into account that $A_{k-1, j}^{n}=\frac{k+n+j-1}{k} A_{k, j}^{n}$, the right-hand side of (3.4) is now given by

$$
M_{k, j}^{n}\left[\begin{array}{c}
u_{k-1-j, j}^{n}(t) \\
v_{k-1-j, j}^{n}(t)
\end{array}\right]=\left[\begin{array}{c}
(n+k+j-1) A_{k, j}^{n} C_{k-j}^{\left(\frac{n-1}{2}+j\right)}(t) \\
A_{k, j}^{n} \sqrt{1-t^{2}} \frac{d}{d t} C_{k-j}^{\left(\frac{n-1}{2}+j\right)}(t)
\end{array}\right]=\left[\begin{array}{c}
u_{k-j, j}^{n}(t) \\
v_{k-j, j}^{n}(t)
\end{array}\right]
$$




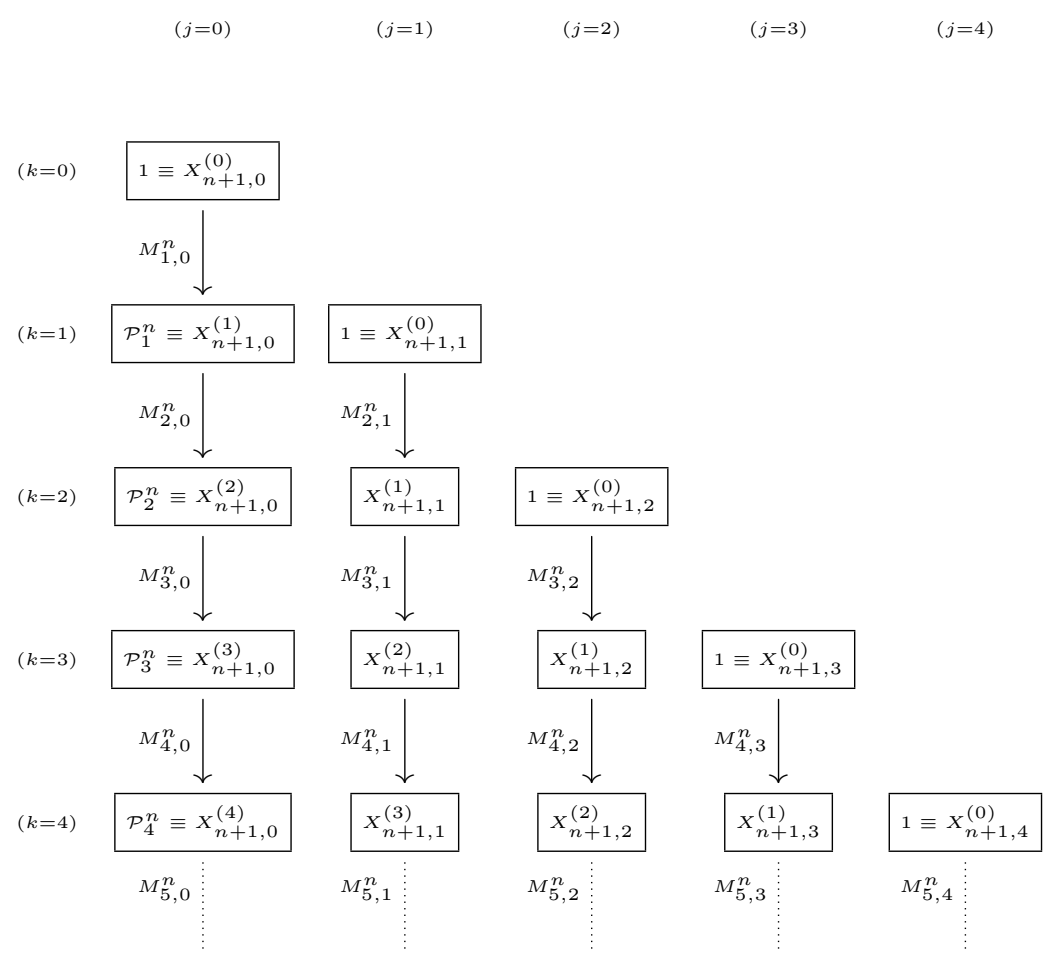

Figure 1: Recurrence scheme

and the theorem is proved.

This theorem can be illustrated by the scheme presented in Figure 1.

Remark 3.1 Notice that for $j=0$, the matrix $M_{k, 0}^{n}$ coincides with the matrix $M_{k}^{n}$ defined in Theorem 3.1. In this case $X_{n+1,0}^{(k)}=\mathcal{P}_{k}^{n}$ and for the complex plane $(n=1), \mathcal{P}_{k}^{1}$ are isomorphic to the holomorphic powers $z^{k}$, where $z=x_{0}+x_{1} e_{1} \in \mathbb{C}$.

With this interpretation for the complex case, Theorem 3.2 describes the multiplicative process of obtaining $z^{k}$ as the product $z . z^{k-1}$ (see [10]).

Remark 3.2 The matrix $M_{k, j}^{n}$ can be decomposed as follows:

$$
M_{k, j}^{n}(t)=D_{k, j}^{n} \mathcal{R}(t),
$$

where

$$
D_{k, j}^{n}:=\left[\begin{array}{cc}
\frac{k}{k-j} & 0 \\
0 & \frac{k}{k-1+n+j}
\end{array}\right] \text { and } \mathcal{R}(t)=\left[\begin{array}{cc}
t & -\sqrt{1-t^{2}} \\
\sqrt{1-t^{2}} & t
\end{array}\right] \text {. }
$$

Introducing polar coordinates $x_{0}=r \cos \theta$ and $|\underline{x}|=r \sin \theta$, where $r=|x|$ and $\theta \in[0, \pi]$, the representation (3.2) corresponds to the representation in polar coordinates of the homogeneous polynomial $X_{n+1, j}^{(k-j)}$. Then $t=\frac{x_{0}}{|x|}=\cos \theta$, and $\sqrt{1-t^{2}}=\sin \theta$. It is now clear that $\mathcal{R}$ is the rotation matrix

$$
\mathcal{R}(\theta)=\left[\begin{array}{cc}
\cos \theta & -\sin \theta \\
\sin \theta & \cos \theta
\end{array}\right]
$$

and the decomposition (3.6) shows the distortion of the rotation process when a multiplication occurs in higher dimensions. Notice that for $n=1$ and $j=0$ (the complex case), the diagonal matrix $D_{k, 0}^{1}$ is the identity. 
Remark 3.3 Recalling that $X_{n+1, k}^{(0)}(x)=1$ for all $x \in \mathcal{A}_{n}$ and for any $k \geq 1$ and each fixed $j(j=0, \ldots, k-1)$, the recursive application of Theorem 3.2 permits to obtain

$$
\begin{aligned}
{\left[\begin{array}{c}
u_{k-j, j}^{n}(t) \\
v_{k-j, j}^{n}(t)
\end{array}\right] } & =M_{k, j}^{n}\left[\begin{array}{l}
u_{k-1-j, j}^{n}(t) \\
v_{k-1-j, j}^{n}(t)
\end{array}\right]=M_{k, j}^{n} M_{k-1, j}^{n}\left[\begin{array}{l}
u_{k-2-j, j}^{n}(t) \\
v_{k-2-j, j}^{n}(t)
\end{array}\right]=\cdots \\
& =M_{k, j}^{n} M_{k-1, j}^{n} \cdots\left[\begin{array}{l}
u_{0}^{n}(t) \\
v_{0}^{n}(t)
\end{array}\right]=\mathcal{M}_{k, j}^{n}\left[\begin{array}{l}
1 \\
0
\end{array}\right],
\end{aligned}
$$

where

$$
\mathcal{M}_{k, j}^{n}:=\prod_{l=0}^{k-j-1} M_{k-l, j}^{n}
$$

In this way, Theorem 3.2 describes a recursive procedure to produce each polynomial $X_{n+1, j}^{(k-j)}(k \geq 0$ and $j=0, \ldots, k-1)$ starting from the initial values 1 and 0 of the scalar and vectorial parts, respectively.

Since

$$
\operatorname{det} M_{k-l, j}^{n}=\frac{(k-l)^{2}}{(k-l-j)(k-l+n+j-1)}, \quad l=0, \ldots, k-j-1,
$$

we have that

$$
\operatorname{det} \mathcal{M}_{k, j}^{n}=\left(\begin{array}{l}
k \\
j
\end{array}\right) \frac{k !}{j !(n+2 j)_{k-j}} \text {. }
$$

\section{Some applications}

From Theorem 3.2 we can derive some results concerning the structure of the building blocks $X_{n+1, j}^{(k-j)}$, for $k \geq 0$ and $j=0, \ldots, k$. The first one is related to the zeros of those polynomials.

Corollary 4.1 For all dimensions $n \geq 1$, for $k \geq 1$ and $j=0, \ldots, k-1, X_{n+1, j}^{(k-j)}(x)=0$ if and only if $x=0$.

Proof For each $k \geq 1$ and a fixed $j(j=0, \ldots, k-1)$, consider $X_{n+1, j}^{(k-j)}$ given by (3.2) and let $x \in \mathcal{A}_{n}$ such that $X_{n+1, j}^{(k-j)}(x)=0$. Then,

$$
|x|^{k-j} u_{k-j, j}^{n}(t)=|x|^{k-j} v_{k-j, j}^{n}(t)=0 .
$$

Using Theorem 3.2 recursively, we obtain

$$
|x|^{k-j} \mathcal{M}_{k, j}^{n}\left[\begin{array}{l}
1 \\
0
\end{array}\right]=\left[\begin{array}{l}
0 \\
0
\end{array}\right] .
$$

Since $\operatorname{det} \mathcal{M}_{k, j}^{n} \neq 0$, it follows at once that last equality holds if and only if $x=0$.

Corollary 4.2 For all dimensions $n \geq 1$, for $k \geq 0$ and a fixed $j(j=0, \ldots, k)$,

$$
\left|X_{n+1, j}^{(k-j)}(x)\right| \leq\left(\begin{array}{c}
k \\
j
\end{array}\right)|x|^{k-j}, x \in \mathcal{A}_{n} .
$$

Proof Using Theorem 3.2 recursively, we have

$$
\left[\begin{array}{l}
u_{k-j, j}^{n}(t) \\
v_{k-j, j}^{n}(t)
\end{array}\right]=\mathcal{M}_{k, j}^{n}\left[\begin{array}{l}
1 \\
0
\end{array}\right]
$$

with $\mathcal{M}_{k, j}^{n}$ given by (3.7). 
The well-known properties of the Euclidean norm in $\mathbb{R}^{2}$ and the corresponding induced matrix norm allow to state that

$$
\left\|\left(u_{k-j, j}^{n}, v_{k-j, j}^{n}\right)\right\|_{2} \leq\left\|\mathcal{M}_{k, j}^{n}\right\|_{2}\|(1,0)\|_{2} \leq \prod_{l=0}^{k-j-1}\left\|M_{k-l, j}^{n}\right\|_{2} .
$$

For each $l(l=0, \ldots, k-j-1)$, the matrix $M_{k-l, j}^{n}\left(M_{k-l, j}^{n}\right)^{T}$ can be written, using decomposition (3.6), as

$$
M_{k-l, j}^{n}\left(M_{k-l, j}^{n}\right)^{T}=D_{k-l, j}^{n} \mathcal{R}(t)(\mathcal{R}(t))^{T}\left(D_{k-l, j}^{n}\right)^{T}=\left(D_{k-l, j}^{n}\right)^{2}
$$

i.e., $M_{k-l, j}^{n}\left(M_{k-l, j}^{n}\right)^{T}$ is the diagonal matrix

$$
M_{k-l, j}^{n}\left(M_{k-l, j}^{n}\right)^{T}=\left[\begin{array}{cc}
\frac{(k-l)^{2}}{(k-l-j)^{2}} & 0 \\
0 & \frac{(k-l)^{2}}{(k-l+n+j-1)^{2}}
\end{array}\right]
$$

It is clear that the largest eigenvalue of this matrix is $\frac{(k-l)^{2}}{(k-l-j)^{2}}$ and therefore $\left\|M_{k-l, j}^{n}\right\|_{2}=\frac{k-l}{k-l-j} \quad l=$ $0, \ldots, k-j-1$. Hence,

$$
\left|X_{n+1, j}^{(k-j)}(x)\right|=|x|^{k-j}\left\|\left(u_{k-j}^{n}, v_{k-j}^{n}\right)\right\|_{2} \leq|x|^{k-j} \prod_{l=0}^{k-j-1} \frac{k-l}{k-l-j}=|x|^{k-j}\left(\begin{array}{l}
k \\
j
\end{array}\right) .
$$

Remark 4.1 Notice that for $j=0$, we obtain the estimate stated in [10] for the polynomials $\mathcal{P}_{k}^{n} \equiv X_{n+1,0}^{(k)}$.

Remark 4.2 An estimate for the building blocks can also be obtained applying the sharp estimate for the Gegenbauer polynomials (see [28])

$$
\left|C_{m}^{\nu}(t)\right| \leq \frac{(2 \nu)_{m}}{m !},|t| \leq 1
$$

to $F_{n+1, j}^{(k-j)}$ in (2.2). It follows then

$$
\left|F_{n+1, j}^{(k-j)}(x)\right| \leq|x|^{k-j}\left(\begin{array}{c}
k \\
j
\end{array}\right)
$$

and therefore, from (2.7),

$$
\left|X_{n+1, j}^{(k-j)}(x)\right| \leq|x|^{k-j}\left(\begin{array}{c}
k \\
j
\end{array}\right)\left(1+\frac{k-j}{n+2 j}\right)
$$

for each fixed $j$.

The estimate for the building blocks obtained in Corollary 4.2 is clearly better and it preserves the sharp estimate (4.1) obtained for the building blocks $F_{n+1, j}^{(k-j)}$ in the harmonic case (2.1).

Corollary 4.3 For each $k \in \mathbb{H}_{0}$, the elements of the orthogonal basis for the space $\mathcal{M}_{k}\left(\mathbb{R}^{n+1}, \mathcal{C} \ell_{0, n}\right)$ given by (2.9) are such that

$$
\left|f_{k, \mu}(x)\right| \leq \frac{k !}{k_{2} ! \prod_{l=2}^{n}\left(k_{l+1}-k_{l}\right) !}|x|^{k}, x \in \mathcal{A}_{n},
$$

where $\mu$ is an arbitrary sequence of integers $\left(k_{n+1}, k_{n}, \ldots, k_{2}\right)$ such that $k=k_{n+1} \geq k_{n} \geq \cdots \geq k_{3} \geq k_{2} \geq 0$ and $k_{2} \in \mathbb{N}_{0}$.

Proof For each $k \geq 0$, from (2.9) we have that

$$
\left|f_{k, \mu}(x)\right|=\prod_{l=2}^{n}\left|X_{l+1, k_{l}}^{\left(k_{l+1}-k_{l}\right)}(x)\right||\zeta|^{k_{2}}, x \in \mathcal{A}_{n},
$$


where $\mu$ is an arbitrary sequence of integers $\left(k_{n+1}, k_{n}, \ldots, k_{2}\right)$ such that $k=k_{n+1} \geq k_{n} \geq \cdots \geq k_{3} \geq k_{2} \geq 0$ and $k_{2} \in \mathbb{N}_{0}$. From the previous corollary and for each $k$ and all sequences of integers $\mu$ the building blocks of the polynomial (2.9) are such that

$$
\left|X_{l+1, k_{l}}^{\left(k_{l+1}-k_{l}\right)}(x)\right| \leq\left(\begin{array}{c}
k_{l+1} \\
k_{l}
\end{array}\right)|x|^{k_{l+1}-k_{l}}, l=2, \ldots, n .
$$

Therefore, taking into account that $|\zeta|^{k_{2}} \leq|x|^{k_{2}}$, for all $x \in \mathcal{A}_{n}$ and $k_{2} \in \mathbb{N}_{0}$, one can write

$$
\left|f_{k, \mu}(x)\right| \leq \prod_{l=2}^{n}\left(\begin{array}{c}
k_{l+1} \\
k_{l}
\end{array}\right)|x|^{k_{l+1}-k_{l}}|\zeta|^{k_{2}} \leq \frac{k !}{k_{2} ! \prod_{l=2}^{n}\left(k_{l+1}-k_{l}\right) !}|x|^{k} .
$$

\section{Acknowledgements}

This work was supported by Portuguese funds through the CIDMA - Center for Research and Development in Mathematics and Applications, the Research Centre of Mathematics of the University of Minho and the Portuguese Foundation for Science and Technology ("FCT - Fundação para a Ciência e a Tecnologia"), within projects PEst-OE/MAT/UI4106/2014 and PEstOE/MAT/UI0013/2014.

\section{References}

[1] P. Appell, Sur une classe de polynômes. Ann. Sci. École Norm. Sup. 9 (1880), no. 2, 119-144.

[2] S. Bock and K. Gürlebeck, On a Generalized Appell System and Monogenic Power Series. Math. Methods Appl. Sci. 33, no. 4, (2010), 394-411.

[3] S. Bock, K. Gürlebeck, Lávisčka, and Souček, V., Gelfand-Tetslin bases for spherical monogenics in dimension 3. Rev. Mat. Iberoam., 28 no. 4, (2012), 1165-1192.

[4] F. Brackx, R. Delanghe, and F. Sommen, Clifford Analysis. Pitman, Boston-London-Melbourne, 1982.

[5] I. Cação, K. Gürlebeck, and H. R. Malonek, Special monogenic polynomials and $L_{2}$-approximation. Adv. Appl. Clifford Algebr., 11 (2001), 47-60.

[6] I. Cação, Constructive Approximation by Monogenic polynomials. Ph.D thesis, Univ. Aveiro, 2004.

[7] I. Cação, K. Gürlebeck, and S. Bock, Complete orthonormal systems of spherical monogenics - a constructive approach. In Methods of Complex and Clifford Analysis, Son LH, Tutschke W, Jain S (eds). SAS International Publications, 2004.

[8] I. Cação, M. I. Falcão, and H. R. Malonek, Laguerre Derivative and Monogenic Laguerre Polynomials: An Operational Approach. Math. Comput. Model. 53, no. 5-6, (2011), 1084-1094.

[9] I. Cação, M. I. Falcão, and H. R. Malonek, On Generalized Hypercomplex Laguerre-Type Exponentials and Applications. In Computational Science and Its Applications - ICCSA 2011, B. Murgante et al.(eds.), Lecture Notes in Computer Science, vol. 6784, Springer-Verlag, Berlin, Heidelberg, (2011), 271-286.

[10] I. Cação, M. I. Falcão, and H. R. Malonek, Matrix representations of a basic polynomial sequence in arbitrary dimension. Comput. Methods Funct. Theory, 12, no. 2, (2012), 371-391.

[11] I. Cação, K. Gürlebeck, and S. Bock, On derivatives of spherical monogenics, Complex Variables and Elliptic Equations, 51, no. 8-11, (2006) 847-869.

[12] I. Cação and H. R. Malonek, Remarks on some properties of monogenic polynomials, In Proceedings of ICNAAM 2006, Simos, T.E. et al. (eds.); Weinheim: Wiley-VCH., (2006) 596-599. 
[13] I. Cação and H. R. Malonek, On Complete Sets of Hypercomplex Appell Polynomials. In AIP Conference Proceedings, Simos, T.E. et al. (eds.) vol. 1048, 2008, 647-650.

[14] B. C. Carlson, Polynomials Satisfying a Binomial Theorem. J. Math. Anal. Appl. 32 (1970), 543-558.

[15] R. Delanghe, F. Sommen, and Souček, V., Clifford algebra and spinor-valued functions. Kluwer Academic Publishers, Dordrecht, 1992.

[16] D. Eelbode, Monogenic Appell sets as representations of the Heisenberg algebra. Adv. Appl. Clifford Algebra, 22, no. 4, (2012), 1009-1023.

[17] M. I. Falcão and H. R. Malonek, Generalized Exponentials Through Appell Sets in $\mathbb{R}^{n+1}$ and Bessel Functions. In AIP Conference Proceedings, Simos, T.E. et al. (eds.), vol. 936, 2007, 738-741.

[18] J.E. Gilbert and M.A.M. Murray, Clifford algebras and Dirac operators in harmonic analysis. Cambridge University Press, Cambridge, 1991.

[19] K. Gürlebeck and H. R. Malonek, A Hypercomplex Derivative of Monogenic Functions in $\mathbb{R}^{n+1}$ and Its Applications. Complex Variables Theory Appl. 39 (1999), 199-228.

[20] K. Gürlebeck, K. Habetha, and W. Sprößig, Holomorphic Functions in the Plane and n-Dimensional Space. Translated from the 2006 German original. Birkhäuser Verlag, Basel, 2008,

[21] W. Hahn, Über die Jacobischen Polynome und zwei verwandte Polynomklassen. Math. Z. 39 (1935), 634-638.

[22] R. Lávička, Canonical Bases for sl(2,c)-Modules of Spherical Monogenics in Dimension 3. Archivum Mathematicum 46 (2010), 339-349.

[23] R. Lávička, Complete Orthogonal Appell Systems for Spherical Monogenics. Complex Anal. Oper. Theory, 6 (2012), 477-489.

[24] H. R. Malonek, A New Hypercomplex Structure of the Euclidean Space $\mathbb{R}^{m+1}$ and the Concept of Hypercomplex Differentiability. Complex Variables 14 (1990), 25-33.

[25] H. R. Malonek, Selected Topics in Hypercomplex Function Theory. In Clifford Algebras and Potential Theory, 7, S.-L. Eriksson (ed.), University of Joensuu, (2004), 111-150.

[26] H. R. Malonek and M. I. Falcão, Special Monogenic Polynomials-Properties and Applications. In AIP Conference Proceedings, Th. E. Simos et al. (eds.) vol. 936, (2007), 764-767.

[27] D. Peña Peña, Shifted Appell Sequences in Clifford Analysis. Results. Math. 63 (2013), 1145-1157.

[28] E. Rainville, Special Functions, Macmillan, New York, 1965. 